\title{
Transdermal Absorption of (-)-Linalool Induces Autonomic Deactivation but has No Impact on Ratings of Well-Being in Humans
}

\author{
Eva Heuberger*,', Sandra Redhammer' and Gerhard Buchbauer' \\ 'Department of Pharmaceutical Chemistry, Center of Pharmacy, University of Vienna, Vienna, Austria
}

\begin{abstract}
Essential lavender oil has a long tradition as a mild sedative in herbal medicine. Relaxing effects after inhalation have also been demonstrated for one of its main constituents, (-)-linalool. The aim of the present investigation was to determine the effects of this monoterpenoid alcohol on human physiological parameters (blood oxygen saturation, breathing rate, eye-blink rate, pulse rate, skin conductance, skin temperature, surface electromyogram as well as systolic and diastolic blood pressure) and assessments of subjective well-being. (-)-Linalool was applied to 14 healthy subjects by percutaneous administration. Inhalation of the fragrance was prevented by means of breathing masks. (-)-Linalool induced deactivation with respect to physiology, that is, a decrease of systolic blood pressure and a smaller decrease of skin temperature, compared to a corresponding control group receiving a placebo, but had no effects on subjective evaluation of well-being.

Neuropsychopharmacology (2004) 29, 1925-1932, advance online publication, 7 July 2004; doi: I 0. I038/sj.npp. 130052 I
\end{abstract}

Keywords: arousal; fragrance; (-)-linalool; physiological monitoring; transdermal administration; well-being

\section{INTRODUCTION}

Nervous disorders, such as states of heightened excitability, anxiety, and stress as well as various forms of sleeping problems are a severe problem in the Western world. As a consequence, prescriptions for sedative drugs, especially tranquillizers and hypnotics of the benzodiazepine receptor agonist type, have increased dramatically (de las Cuevas et al, 2003; Hirst and Sloan, 2002). Since these drugs are associated with serious adverse effects, for example, rebound and hang-over effects, reduced alertness and efficiency during the day as well as physical and psychic addiction, chronic intake of these substances is somewhat problematic. Recent studies demonstrated that herbal preparations containing lemon balm (Melissa officinalis, L.) (Ballard et al, 2002; Kennedy et al, 2003), valerian (Valeriana officinalis, L.) (Cerny and Schmid, 1999) or St John's wort (Kommission E Monograph, 1989) proved as alternatives with satisfying efficacy and tolerability. Also lavender (Lavandula angustifolia, Mill.) is highly appre-

\footnotetext{
*Correspondence: Dr E Heuberger, Department of Pharmaceutical Chemistry, Center of Pharmacy, University of Vienna, Althanstrasse 14, A- 1090 Vienna, Austria, Tel: + 43 | 4277 55I60, Fax: + 43 I 4277 955 I, E-mail: eva.heuberger@univie.ac.at

Received 27 October 2003; revised 20 April 2004; accepted 4 June 2004

Online publication: 9 June 2004 at http://www.acnp.org/citations/ Npp06090403494/default.pdf
}

ciated in herbal medicine because of its carminative and spasmolytic properties (Lis-Balchin and Hart, 1999; Cavanagh and Wilkinson, 2002). The essential oil, predominantly found in the flowers, is used as a mild sedative in phytoand aromatherapy due to its calming, stress-relieving and relaxing effects after inhalation which have been substantiated in both animal (Buchbauer et al, 1993; Bradshaw et al, 1998) and human studies (Schulz et al, 1998; Torii et al, 1988).

Since the 1980s, a considerable number of investigations has been carried out to evaluate activating and sedative effects of a variety of essential oils and odorants after inhalation using neurophysiological as well as behavioral measures. In contrast, stimulating and relaxing effects of fragrances after transdermal absorption have hardly been assessed. Studies recently performed by our group (Hongratanaworakit et al, 2000, 2004) showed that the route of administration crucially influenced the effects observed for the essential oil of East Indian Sandalwood and its main constituent $\alpha$-santalol. After inhalation, the odorants induced activation of healthy human subjects in terms of both physiological and behavioral parameters, whereas after transdermal administration, the same fragrances elicited a decrease of physiological arousal. These findings may be explained by the effectiveness of different mechanisms of action when odorants are applied by means of inhalation as opposed to percutaneous absorption. 
(-)-Linalool which is one of the major compounds of essential lavender oil was found to possess sedative effects after inhalation both in animals (Elisabetsky et al, 1995) and in man (Hardy et al, 1995; Sugawara et al, 1998). Therefore, in this study, we were interested in the effects of (-)linalool on physiological parameters and on the subjective evaluation of well-being in humans after transdermal administration.

\section{MATERIALS AND METHODS}

In total, 32 healthy subjects ( 13 males, 19 females) with normal body mass indices (mean $20.75 \pm 2.42$ ) aged between 20 and 34 years (mean age $24.0 \pm 2.6$ years) were tested in individual sessions. They were recruited by advertisement at the University of Vienna and were reimbursed for their participation. In all, 18 subjects were assigned to the control group while the remaining 14 subjects formed the experimental group. At $48 \mathrm{~h}$ prior to testing, subjects had to abstain from food, beverages, and toiletries containing (-)linalool, as well as from tea, coffee, and alcohol. Subjects were fully briefed, gave written informed consent to all aspects of the study, and were free to withdraw at any time. The study was approved by the Viennese Ethic Commission (permissions no. 324/96, 419/98).

One session consisted of two trials of $20 \mathrm{~min}$ each. At the beginning as well as at the end of each trial, ratings of subjective well-being were assessed on visual analogue scales (VAS). Physiological parameters were recorded continuously during each trial. Blood pressure was measured at the beginning as well as at the end of each trial. In the first trial, which served as a control for influences of the experimental setup, peanut oil was administered to all subjects. In the second trial, subjects in the control group again received peanut oil, whereas (-)-linalool was applied in the experimental group.

(-)-Linalool was obtained from Dragoco GmbH (now Symrise), Vienna. In the experimental group, $1 \mathrm{ml}$ of a $10 \%$ (v/v) solution of the fragrance in peanut oil (Arachidis oleum, Pharmacopoea Europaea, Monograph, 1997) was applied to the lower abdomen and subjects were asked to massage until most of the oil had been absorbed by the skin. To prevent evaporation of (-)-linalool, the area was then covered with plastic film. In the control group, $1 \mathrm{ml}$ of pure peanut oil was used per subject. In all groups, subjects were supplied with pure air by means of breathing masks in order to prevent any olfactory stimulation.

Seven physiological parameters were recorded simultaneously and in real time by means of MP100WSW hardware (Biopac Systems, Inc., Santa Barbara, California, USA) and Acqknowledge ${ }^{\circledR}$ software (V3.2.6, (C) 1992-1997, Biopac Systems, Inc., Santa Barbara, California, USA). Sampling rate was $100 \mathrm{~Hz}$.

Blood oxygen saturation (BOS) and pulse rate (PR) were measured with a pulse oximeter module (OXY100A) and a photoelectrical sensor (BIOPAC-TSD123B). The noninvasive transducer was placed on the first phalanx of the ring finger of the nondominant hand with noncaustic adhesive tape (Biopac Systems Inc., 1999; Stern et al, 2001).

Breathing rate (BR) was assessed using a SKT100B amplifier and a TSD102D surface temperature thermistor probe, which registers breathing cycles on the basis of the difference in temperature between inhaled and exhaled air. The probe was placed at the entrance of the left nostril with noncaustic adhesive tape (Stern et al, 2001).

Eye-blinks (EB) were recorded by means of a EOG100B amplifier, $\mathrm{Ag} / \mathrm{AgCl}$ surface electrodes (EL503), and adhesive disks (ADD204). Two electrodes, which were filled with conductive gel, were placed over the orbicularis oculi muscle on a vertical line (Stern et al, 2001).

Skin conductance (SC) was recorded using a GSR100B amplifier and $\mathrm{Ag} / \mathrm{AgCl}$ finger electrodes (BIOPACTSD103A) via the constant voltage $(0.5 \mathrm{~V})$ technique. Electrodes were filled with conductive gel and placed on the second phalanx of the middle and the index finger of the nondominant hand with noncaustic adhesive tape. Electrode positioning was in compliance with traditional recommendations (Fowles et al, 1981).

Skin temperature (ST) was measured using a SKT100B amplifier and a fast response thermistor (BIOPACTSD102A). The sensor was placed on the middle of the back of the nondominant hand with noncaustic adhesive tape.

Surface electromyogram (EMG) was recorded with a EMG100B amplifier, Ag/AgCl surface electrodes (EL208S) and adhesive disks (ADD208). EMG activity was recorded by placing two electrodes, which were filled with conductive gel, over the Splenius capitis muscle (Stern et al, 2001).

In addition, systolic and diastolic blood pressure (SBP and DBP) were registered in the dominant arm by sphygmomanometry using an automated system (Hartmann Digital HG160, Paul Hartmann AG, D-89522 Heidenheim) four times throughout the experiment (Stern et al, 2001).

VAS adopted from the 'Bf-S, Befindlichkeitsskala' (wellbeing scale) (von Zerssen and Koeller, 1976) were used to obtain ratings of subjective well-being. They were presented four times throughout the experiment, that is, at the beginning and at the end of each trial, and consisted of $100 \mathrm{~mm}$ lines for the items 'relaxation', 'vigor', 'calmness', 'attentiveness', 'mood,' and 'alertness'. Subjects were asked to mark their feelings for each item between the two possible extremes: relaxed (on the left) and tense (on the right) for the item 'relaxation', vigorous (on the left) and feeble (on the right) for the item 'vigor', calm (on the left) and restless (on the right) for the item 'calmness', attentive (on the left) and inattentive (on the right) for the item 'attentiveness', cheerful (on the left) and bad tempered (on the right) for the item 'mood', and alert (on the left), and tired (on the right) for the item 'alertness'.

\section{Procedure}

All experiments were conducted in a bright and quiet room. Ambient temperature was $21-24^{\circ} \mathrm{C}$. Subjects were seated in a semireclined position, providing easy access to attach the electrodes. They were informed that a fragrance would be administered, but did not know which and when.

Subjects were interviewed about their personal data, that is, name, age, sex, weight, and height and about their dominant hand by means of the Edinburgh Handedness Inventory (Oldfield, 1971). Subsequently, they were informed about the proceedings. Then, SBP and DBP were measured. After the electrodes had been attached, the 
breathing mask was attached comfortably to the subject's face to cover nose and mouth. The air flow from the tank was adjusted individually. In order to prevent perception of any odor present in the experimental chamber, the valve of the tank was opened until air was flowing out of the exhaust tube of the mask even when subjects were deeply inhaling. Following, $1 \mathrm{ml}$ of peanut oil was applied to the skin of the lower abdomen, and subjects were asked to massage it into their skin for about $2 \mathrm{~min}$. As soon as the oil had been absorbed by the skin the area was covered with plastic film, subjects were asked to rate their subjective well-being and the recording of the physiological parameters was started. After completion of the first trial, the residues of the fatty oil were eliminated, subjects were allowed to remove the breathing mask and the rating scales were presented. SBP and DBP were measured at the end of the first trial. This procedure was repeated in the second trial, which followed consecutively with two exceptions: at the beginning of the second trial, blood pressure was registered after the presentation of the rating scales and subjects in the experimental group received $1 \mathrm{ml}$ of the (-)-linalool solution. At the end of the session, subjects were asked if they had perceived an odor during any trial. None of the subjects indicated to have perceived any odor different than that of the mask.

\section{Data Reduction}

The physiological parameters were computed by means of AcqKnowledge ${ }^{\circledR}$ software. Each trial was divided into four intervals of $5 \mathrm{~min}$. Individual mean values of ST, level of SC (SCL), PR, and BOS within each interval were obtained for every subject. BR was determined by counting the number of breaths per interval and was subsequently converted into breaths per minute. Similarly, eye-blink rate (EBR) was determined by counting the number of eye-blinks per minute. The raw EMG signals were converted to the root mean square (rms) values.

For each subject and every parameter, the mean value in a given interval (eg the first) in the second trial was subtracted from the mean value in the corresponding interval (ie the first) in the first trial, resulting in four individual differences. These were averaged to give one individual intertrial difference. With respect to SPB and DBP, individual difference scores were calculated between measurements.

Regarding self-ratings, on each scale, the distance of the mark from the left-hand side was measured in millimeters. Individual difference scores between ratings were calculated for each item.

\section{Statistics}

Systat ${ }^{\circledR} 9.0$ (Systat Software Inc., Richmond, California, USA, (C) 1999) was used for statistical analysis. The effects of (-)-linalool on the physiological parameters and ratings of subjective well-being were determined by comparing the differences between the control group and the experimental group by means of Mann-Whitney $U$-test and KruskalWallis one-way analysis of variance.

\section{RESULTS}

\section{Systolic (SBP) and Diastolic Blood Pressure (DBP)}

Medians and mean interquartile ranges (MIQR) of SBP and DBP on all measurements for the control group and the (-)-linalool group are presented in Table 1. Medians and MIQR of the differences between measurements of SBP and DBP for the control group and the experimental group are shown in Figures 1 and 2, respectively. At the beginning of the second trial compared with the end of the first trial (difference 2/3) SBP significantly increased (negative difference) in the (-)-linalool group, while it hardly changed in the control group $(U=160.5$, $P=0.026)$. In contrast, at the end of trial 2 compared with the beginning of that trial (difference 3/4) SBP significantly decreased in the (-)-linalool group, whereas an increase was found in the control group $(U=42.5$, $P=0.005$ ). For the other differences between measurements, no significant differences were observed between groups $(P>0.1)$.

At the beginning of the second trial compared with the beginning of the first trial (difference 1/3) a trend towards a larger decrease of DBP (positive difference) in the control group than in the (-)-linalool group was found $(U=147.0$, $P=0.098)$. At the beginning of trial 2 compared with the end of trial 1 (difference 2/3) the decrease of DBP in the control group was in significant contrast to the increase in the (-)-linalool group $(U=151.0, P=0.030)$. No significant differences between groups were found for the other differences between measurements $(P>0.1)$.

\section{Skin Temperature (ST) and Skin Conductance Level (SCL)}

Medians and MIQR of ST and SCL in the first and the second trial as well as medians and MIQR of the overall intertrial differences of ST and SCL for the control group and the (-)-linalool group are presented in Table 1 . Medians and MIQR of ST and SCL in the single $5 \mathrm{~min}$ intervals in the first and the second trial for both groups are given in Table 2. Medians and MIQR of the differences of ST and SCL in the single $5 \mathrm{~min}$ intervals for the control group and the (-)-linalool group are depicted in Figure 3. ST decreased more pronouncedly in the control group than in the (-)-linalool group, resulting in a significantly larger positive difference in the control group than in the $(-)$ linalool group $(U=181.0, P=0.037)$. While the differences did not differ significantly between groups in the first interval $(P>0.1)$, trends towards a larger decrease of ST in the control group compared with the (-)-linalool group were observed in the second $(U=177.0, P=0.053)$, the third $(U=175.0, P=0.063)$ and fourth interval $(U=171.0$, $P=0.087)$.

No significant differences between the control group and the (-)-linalool group were found for the overall intertrial differences of SCL $(P>0.1)$. Trends towards a larger increase of SCL in the (-)-linalool group as compared to the control group were found in the first $(U=175.0$, $P=0.063)$ and third interval $(U=161.0, P=0.095)$. The differences in the second and fourth interval did not differ significantly $(P>0.1)$. 
Table I Medians and Mean Interquartile Ranges (MIQR) of Systolic Blood Pressure (SBP), Diastolic Blood Pressure (DBP), Skin Temperature (ST), Subjective Attentiveness, Subjective Mood, EyeBlink Rate (EBR) and Pulse Rate (PR) for the Control Group (C) and the (-)-Linalool Group $(L)$

\begin{tabular}{|c|c|c|c|c|}
\hline & \multicolumn{2}{|c|}{ Median } & \multicolumn{2}{|c|}{ MIQR } \\
\hline & C & $\mathbf{L}$ & C & $\mathbf{L}$ \\
\hline \multicolumn{5}{|l|}{ SBP } \\
\hline Measurement I & 122.00 & 133.00 & 7.00 & 11.63 \\
\hline Measurement 2 & I 15.00 & 130.00 & 6.00 & 10.50 \\
\hline Measurement 3 & 116.50 & 129.00 & 5.50 & 7.25 \\
\hline Measurement 4 & $1 \mid 8.00$ & 128.00 & 5.50 & 11.00 \\
\hline \multicolumn{5}{|l|}{$D B P$} \\
\hline Measurement I & 76.00 & 81.00 & 3.50 & 4.50 \\
\hline Measurement 2 & 70.00 & 72.00 & 2.50 & 12.63 \\
\hline Measurement 3 & 70.00 & 79.50 & 10.88 & 8.25 \\
\hline Measurement 4 & 118.00 & 128.00 & 4.00 & 6.38 \\
\hline \multicolumn{5}{|l|}{ ST } \\
\hline Trial I & 31.74 & 32.38 & 1.70 & 0.78 \\
\hline Trial 2 & 31.14 & 32.40 & 2.04 & 0.81 \\
\hline Intertrial difference & 0.59 & 0.08 & 0.42 & 0.37 \\
\hline \multicolumn{5}{|l|}{$S C L$} \\
\hline Trial I & 2.95 & 1.85 & 0.85 & 0.99 \\
\hline Trial 2 & 2.87 & 2.39 & 1.22 & 1.28 \\
\hline Intertrial difference & -0.13 & -0.35 & 0.25 & 0.24 \\
\hline \multicolumn{5}{|l|}{ Attentiveness } \\
\hline Rating I & 21.50 & 19.00 & 9.00 & 11.50 \\
\hline Rating 2 & 26.75 & 33.00 & 8.50 & 20.50 \\
\hline Rating 3 & 31.75 & 26.00 & 9.00 & 9.50 \\
\hline Rating 4 & 35.00 & 26.00 & 10.75 & 13.00 \\
\hline \multicolumn{5}{|l|}{ Mood } \\
\hline Rating I & 22.00 & 18.00 & 7.00 & 12.50 \\
\hline Rating 2 & 21.50 & 36.50 & 5.25 & 18.50 \\
\hline Rating 3 & 24.00 & 23.00 & 6.75 & 11.00 \\
\hline Rating 4 & 22.50 & 22.50 & 6.25 & 10.50 \\
\hline \multicolumn{5}{|l|}{$E B R$} \\
\hline Trial I & $|6.6|$ & 6.90 & 7.27 & 8.68 \\
\hline Trial 2 & 16.03 & 11.22 & 6.10 & 2.15 \\
\hline Intertrial difference & -0.40 & -2.33 & 2.43 & 3.58 \\
\hline \multicolumn{5}{|l|}{$P R$} \\
\hline Trial I & 69.90 & 69.97 & 7.52 & 5.30 \\
\hline Trial 2 & 67.45 & 68.09 & 4.74 & 3.84 \\
\hline Intertrial difference & 1.84 & 2.87 & 1.47 & 1.48 \\
\hline
\end{tabular}

Neuropsychopharmacology

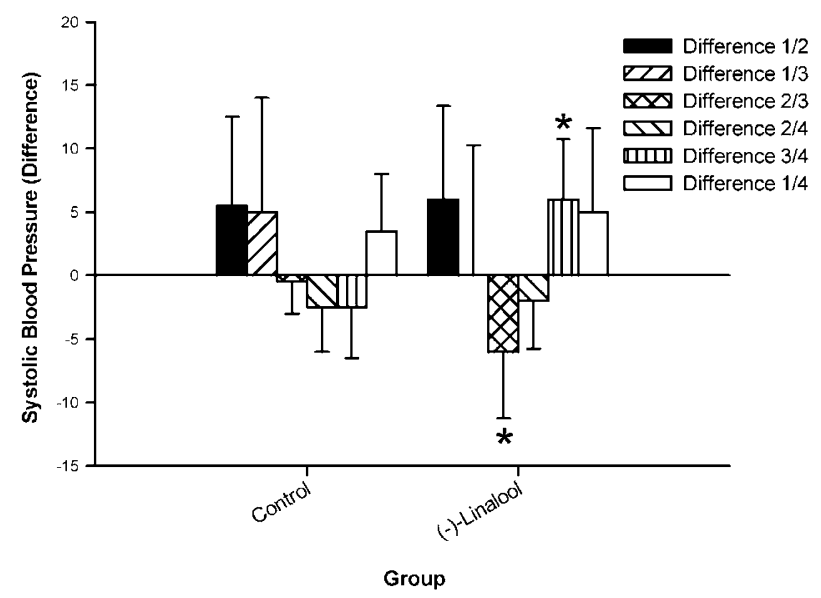

Figure I Medians and mean interquartile ranges of systolic blood pressure differences in the control group and the (-)-linalool group; difference I/2: difference between measurements I and 2, difference I/3: difference between measurements 1 and 3, difference 2/3: difference between measurements 2 and 3 , difference 2/4: difference between measurements 2 and 4, difference 3/4: difference between measurements 3 and 4, difference 1/4: difference between measurements 1 and 4 ; *significant $(P \leqslant 0.05)$ difference between control group and $(-)$-linalool group.

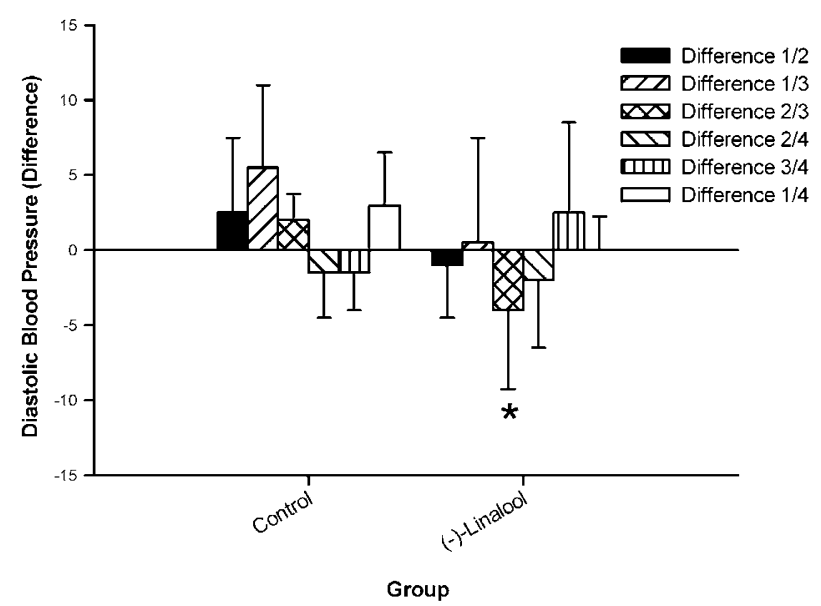

Figure 2 Medians and mean interquartile ranges of diastolic blood pressure differences in the control group and the (-)-linalool group; difference 1/2: difference between measurements I and 2, difference I/3: difference between measurements 1 and 3 , difference 2/3: difference between measurements 2 and 3, difference 2/4: difference between measurements 2 and 4, difference 3/4: difference between measurements 3 and 4, difference 1/4: difference between measurements 1 and 4 *significant $(P \leqslant 0.05)$ difference between control group and $(-)$-linalool group.

\section{Eye-Blink Rate (EBR) and Pulse Rate (PR)}

Medians and MIQR of EBR and PR in the first and the second trial as well as medians and MIQR of the overall intertrial differences of EBR and $\mathrm{PR}$ for the control group and the (-)-linalool group are shown in Table 1. Medians and MIQR of EBR and PR in the single 5 min intervals in trials 1 and 2 for both groups are presented in Table 2. The medians and MIQR of the differences of EBR and PR in the 
Table 2 Medians and Mean Interquartile Ranges (MIQR) of Skin Temperature (ST), Skin Conductance Level (SCL), Eye-Blink Rate (EBR) and Pulse Rate (PR) in Each 5 min Interval of Trials I and 2 for the Control Group (C) and the (-)-Linalool Group (L)

\begin{tabular}{|c|c|c|c|c|c|c|c|c|}
\hline \multirow[b]{2}{*}{ Interval } & \multicolumn{2}{|c|}{$\begin{array}{l}\text { Median } \\
\text { (trial I) }\end{array}$} & \multicolumn{2}{|c|}{$\begin{array}{c}\text { MIQR } \\
\text { (trial I) }\end{array}$} & \multicolumn{2}{|c|}{$\begin{array}{c}\text { Median } \\
\text { (trial 2) }\end{array}$} & \multicolumn{2}{|c|}{$\begin{array}{c}\text { MIQR } \\
\text { (trial 2) }\end{array}$} \\
\hline & C & $\mathbf{L}$ & C & $\mathbf{L}$ & C & $\mathbf{L}$ & C & $\mathbf{L}$ \\
\hline \multicolumn{9}{|l|}{ ST } \\
\hline I & 31.59 & 32.17 & 1.57 & 0.70 & 31.51 & 32.25 & 1.65 & 0.89 \\
\hline 2 & 31.86 & 32.43 & 1.67 & 0.76 & 31.24 & 32.59 & 2.03 & 0.74 \\
\hline 3 & 31.75 & 32.49 & 1.69 & 0.71 & 31.04 & 32.46 & 2.12 & 0.92 \\
\hline 4 & 31.85 & 32.49 & 1.70 & 0.66 & 30.78 & 32.32 & 2.06 & 0.91 \\
\hline \multicolumn{9}{|l|}{$S C L$} \\
\hline I & 3.76 & 2.58 & 0.87 & 0.89 & 3.45 & 3.13 & 0.90 & 1.07 \\
\hline 2 & 2.54 & 1.79 & 0.97 & 0.82 & 2.81 & 2.07 & 1.13 & 1.19 \\
\hline 3 & 2.52 & 1.57 & 1.01 & 1.01 & 2.51 & 1.96 & 1.34 & 1.26 \\
\hline 4 & 2.49 & 1.48 & 0.98 & 1.14 & 2.32 & 2.13 & 1.40 & 1.18 \\
\hline \multicolumn{9}{|l|}{$E B R$} \\
\hline I & 18.78 & 7.50 & 9.80 & 8.40 & 17.70 & 10.80 & 9.60 & 6.30 \\
\hline 2 & 16.00 & 4.50 & 6.80 & 8.00 & 14.50 & 11.70 & 5.80 & 4.80 \\
\hline 3 & 15.70 & 6.80 & 6.60 & 9.30 & 17.00 & 12.20 & 7.80 & 5.10 \\
\hline 4 & 14.80 & 9.10 & 8.30 & 8.50 & 16.10 & 10.00 & 7.90 & 6.50 \\
\hline \multicolumn{9}{|l|}{$P R$} \\
\hline 1 & 69.51 & 71.73 & 7.90 & 3.72 & 68.68 & 69.12 & 6.08 & 3.80 \\
\hline 2 & 70.74 & 70.36 & 8.41 & 4.17 & 69.18 & 67.68 & 5.03 & 4.28 \\
\hline 3 & 69.20 & 70.66 & 7.73 & 6.69 & 67.30 & 66.78 & 5.26 & 4.08 \\
\hline 4 & 69.50 & 68.67 & 7.26 & 5.75 & 66.73 & 66.76 & 4.18 & 3.86 \\
\hline
\end{tabular}

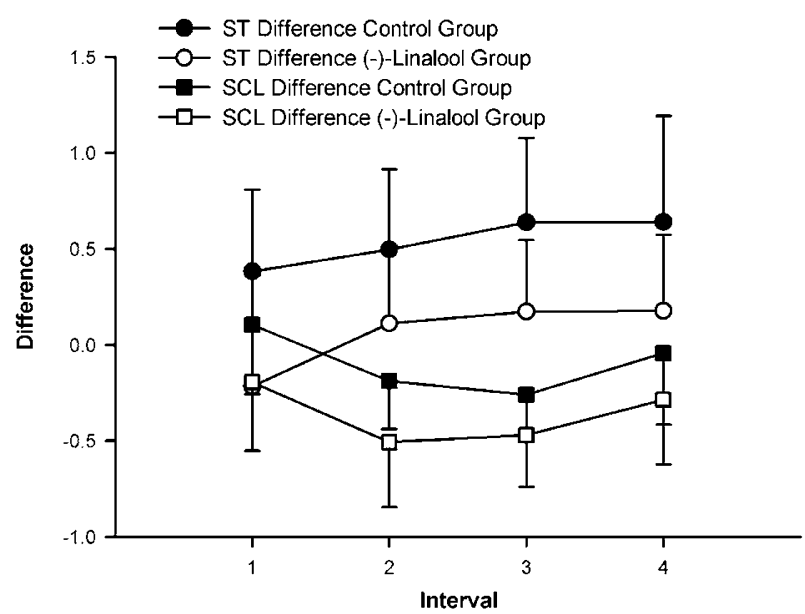

Figure 3 Medians and mean interquartile ranges of skin temperature (ST) and skin conductance level (SCL) differences in each 5 min interval in the control group and the (-)-linalool group.

single $5 \mathrm{~min}$ intervals for the control group and the experimental group are depicted in Figure 4. The overall intertrial differences of EBR did not differ significantly

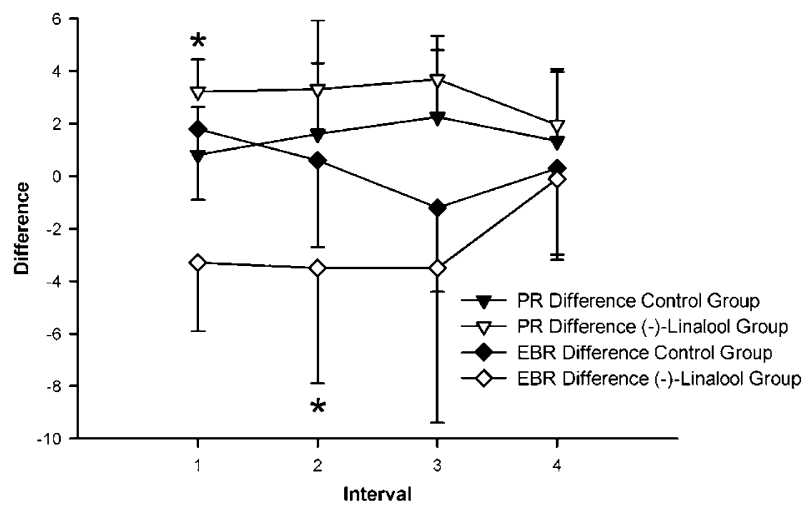

Figure 4 Medians and mean interquartile ranges of pulse rate $(P R)$ and eye-blink rate (EBR) differences in each 5 min interval in the control group and the $(-)$-linalool group; *significant $(P \leqslant 0.05)$ difference between control group and (-)-linalool group.

between the control group and the (-)-linalool group $(P>0.1)$. A trend towards a larger increase of EBR in the (-)-linalool group in contrast to the control group was found in the first interval $(U=170.0, P=0.095)$. In the second interval, the difference between groups was significant $(U=179.0, P=0.044)$. However, in the third and fourth interval, no significant differences were observed between groups $(P>0.1)$.

For the overall intertrial differences of PR, no significant differences were revealed between the control group and the $(-)$-linalool group $(P>0.1)$. A significantly larger decrease of PR in the (-)-linalool group in contrast to the control group was revealed in the first interval $(U=74.0, P=0.048)$. A trend towards the same direction was observed in the third interval $(U=77.0, P=0.077)$. The differences in the second and fourth interval did not differ significantly between groups $(P>0.1)$.

\section{Attentiveness and Mood}

Medians and MIQR of subjective attentiveness and mood on all ratings for the control group and the (-)-linalool group are given in Table 1. Medians and MIQR of the differences between ratings of subjective attentiveness and mood for both groups are presented in Figures 5 and 6, respectively. Subjects in the (-)-linalool group felt significantly more attentive (positive difference) than those in the control group both at the beginning and at the end of the second trial compared to the end of trial 1 (difference 2/3: $U=59.0$, $P=0.011$; difference $2 / 4: U=53.5, P=0.006$ ). The other differences between ratings of attentiveness did not differ significantly between groups $(P>0.1)$.

Subjects in the (-)-linalool group felt significantly more cheerful (positive difference) than those in the control group both at the beginning and at the end of the second trial compared to the end of the first trial (difference 2/3: $U=61.0, \quad P=0.013$; difference 2/4: $U=68.5, P=0.029)$. No significant differences between groups were found for the other differences between ratings of mood $(P>0.1)$. 


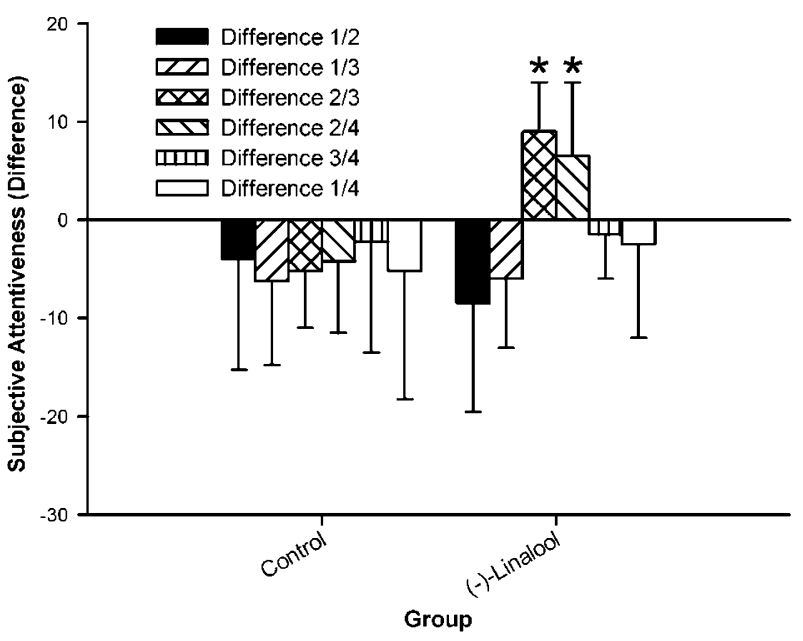

Figure 5 Medians and mean interquartile ranges of subjective attentiveness differences in the control group and the (-)-linalool group; difference I/ 2 : difference between ratings 1 and 2 , difference I/3: difference between ratings I and 3, difference $2 / 3$ : difference between ratings 2 and 3 , difference 2/4: difference between ratings 2 and 4 , difference 3/4: difference between ratings 3 and 4, difference I/4: difference between ratings I and 4; *significant $(P \leqslant 0.05)$ difference between control group and $(-)$-linalool group.

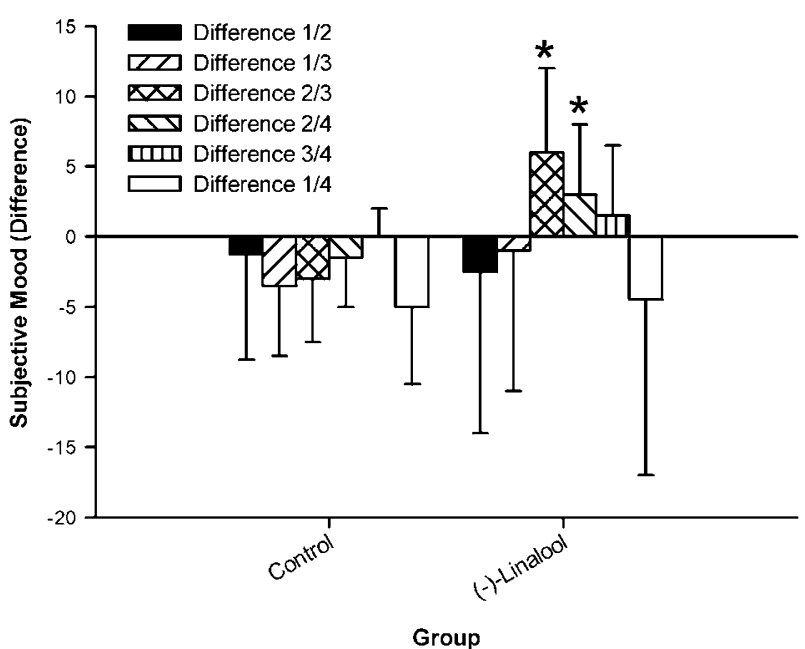

Figure 6 Medians and mean interquartile ranges of subjective mood differences in the control group and the (-)-linalool group; difference I/2: difference between ratings 1 and 2, difference 1/3: difference between ratings I and 3, difference 2/3: difference between ratings 2 and 3 , difference $2 / 4$ : difference between ratings 2 and 4, difference 3/4: difference between ratings 3 and 4, difference I/4: difference between ratings I and 4; *significant $(P \leqslant 0.05)$ difference between control group and $(-)$-linalool group.

Blood Oxygen Saturation (BOS), Breathing Rate (BR), Surface-Electromyogram (EMG), Subjective Relaxation, Vigor, Calmness, and Alertness

No significant effects of (-)-linalool were observed on these parameters $(P>0.1$, data not shown).

\section{DISCUSSION}

The present investigation aimed at the determination of the effects of (-)-linalool on seven physiological parameters which, with the exception of cross-striate muscle activity, are controlled by the autonomic nervous system, and on ratings of subjective well-being after percutaneous absorption. In line with reports from the literature which emphasize the stress-relieving, relaxing, and sedating effects of the essential oil of lavender (Buchbauer et al, 1991; Kerl, 1997; Yagyu, 1994) containing (-)-linalool as a major compound as well as the sedative, hypnotic, anticonvulsant, and local anesthetic actions of (-)-linalool (Elisabetsky et al, 1999; Re et al, 2000; Sugawara et al, 1998) transdermal administration of (-)-linalool was expected to lower both physiological and behavioral arousal levels in human subjects.

In our study, (-)-linalool seemed to exert deactivating effects with respect to physiology, but did not show any effects on subjective evaluation of well-being. At the physiological level, systolic blood pressure increased in the experimental group at the beginning of the second trial, that is, immediately after (-)-linalool had been administered to the subjects, while it decreased in the control group. This result indicates a higher arousal level at the beginning of the second trial in subjects in the experimental group rather than an effect of the fragrance. After the application of (-)-linalool, that is, at the end of the second trial, systolic blood pressure significantly decreased in the experimental group, which may be due to a deactivating effect of (-)linalool. Also, the results for diastolic blood pressure - which decreased less pronouncedly in the (-)-linalool group when the beginnings of the two trials were compared and even increased at the onset of the second in comparison to the end of the first trial-suggest different activation levels in the control group and the experimental group at the beginning of the second trial. In addition, these data again point towards a deactivating effect of the fragrance. Although comparison of the differences between the end of the second trial and the beginning of the same trial did not result in a significant contrast between the experimental group and the control group, it may be speculated that (-)linalool reduced the initially heightened activation level in the experimental group. Visual inspection of the data showed a similar pattern for diastolic as for systolic blood pressure, that is, a decrease of diastolic blood pressure in the (-)-linalool group and an increase in the control group, and thus supports this interpretation. Administration of the fragrance led to a significantly smaller decrease of skin temperature than administration of pure peanut oil. This finding suggests a smaller increase in sympathetic tone, that is, activation, in the (-)-linalool group, since the sympathetic division of the ANS controls skin temperature via the tonicity of the cutaneous vascular walls. Analysis of the differences in the single 5 min intervals revealed a trend towards this direction from the second interval on. Jaeger et al (1992) reported that traces of (-)-linalool could be detected in human blood samples 5 min after the application of a massage oil containing $2 \%$ of essential lavender oil. Taking into account these pharmacokinetic data it seems likely that any effects of (-)-linalool should not show earlier than $5 \mathrm{~min}$ after the onset of administration.

The results of eye-blink rate could also be indicative of an initially higher arousal level in the experimental group, which was probably lowered by (-)-linalool since differences between groups were observed at the beginning of the 
second trial but vanished in the course of this trial. The results for heart rate are somewhat ambiguous. In the experimental group, heart rates decreased significantly more pronouncedly than in the control group in the first interval of the second as compared to the first trial. A trend towards the same direction was found in the third $5 \mathrm{~min}$ interval, but no significant differences were revealed between groups in the second and the last interval. With respect to the above-mentioned pharmacokinetic properties of (-)-linalool this early decrease of heart rate was very unlikely evoked by the fragrance. Similar to the findings for eye-blink rate it rather seems to indicate a difference between the physiological activation levels of the control group and the experimental group at the beginning of the second trial. However, the decrease of heart rate suggests a lower arousal level in the experimental group, which is contradictory to the results for eye-blink rate. On the other hand, a decrease of heart rate is associated with activation processes, especially in tasks involving attention to and processing of external stimuli (Schandry, 1989; Velden, 1994). In this context, heart rate decreases are accompanied by increases of blood pressure (Velden, 1994). As mentioned above, in our study systolic blood pressure initially increased in subjects in the (-)-linalool group. However, in the experimental setting of this investigation, subjects were neither instructed to attend to any stimuli nor did they tell to have perceived any odor so that an interpretation in terms of increased arousal hardly holds for the heart rate data. Visual inspection of the raw data showed that in the first interval of the first trial heart rate in the (-)-linalool group was higher than in the control group and that it decreased quite drastically during the first trial in the experimental group, while it remained relatively stable in the control group. In contrast, heart rates in the second trial seemed to be in comparable ranges in both groups. Probably, the observed decrease of heart rate in the (-)linalool group at the beginning of the second trial was due to a difference in baseline values. Statistical analysis of heart rate in the first trial, however, did not reveal any differences between the control group and the experimental group. Thus, this finding remains unclear.

Subjects in the (-)-linalool group felt significantly more attentive and more cheerful than those in the control group at the beginning of the second trial, that is, immediately after the application of the odorant, compared with the end of the baseline trial. These findings signify a higher activation level in the experimental group than in the control group also in terms of well-being. In contrast to physiological arousal the behavioral activation level in the (-)-linalool group remained unchanged after the fragrance had been applied for $20 \mathrm{~min}$. This is demonstrated by significantly higher ratings of subjective attentiveness and mood also at the end of the second trial compared to the end of the first trial as well as by the lack of significant between-group differences for the comparison of ratings at the end and at the beginning of the second trial. Moreover, none of the other items was influenced by (-)-linalool. These findings suggest that (-)-linalool had no effects on subjective ratings of well-being. In contrast to our findings, inhalation of essential lavender oil has been reported to improve mood ratings in healthy subjects (Knasko, 1992; Diego et al, 1998). These effects might be due to psychological effects triggered by qualitative aspects of odor information (Jellinek, 1997; Kirk-Smith, 1995) and are thus inactive when olfactory processing is prevented.

It should not be left unmentioned that we do not know why subjects in the experimental group were more aroused at the beginning of the second trial than subjects in the control group, neither can we rule out that this increased arousal level in the experimental group would have returned to the baseline level also under placebo conditions. On the other hand, there is no satisfying explanation why ANS, but not behavioral arousal levels should decrease under such conditions. Thus, we favor the interpretation of our results in terms of a physiologically deactivating effect of (-)linalool, while there was no such effect on subjective wellbeing in healthy human subjects.

Percutaneous administration of fragrances is a valuable and promising tool in the evaluation of the effects of odorant substances on humans since it offers the opportunity to study these effects without the implication of other odor-related factors, such as a person's hedonic preferences or subjective experience in the past. In agreement with previous reports our study demonstrates a deactivating effect of $(-)$-linalool on human subjects after transdermal absorption.

\section{ACKNOWLEDGEMENTS}

We thank Dragoco GmbH (now Symrise), Vienna, for financial support of this study.

\section{REFERENCES}

Ballard CB, O’Brien JT, Reichelt K, Perry E (2002). Aromatherapy as a safe and effective treatment for the management of agitation in severe dementia: the result of a double-blind, placebocontrolled trial with melissa. J Clin Psychiatry 63: 553-558.

Biopac Systems Inc (1999). MP100 System Guide, Reference Manual Version 3.5.0 for MP100 Hardware and AcqKnowledge ${ }^{\mathbb{R}}$ Software. Biopac Systems, Inc.: Santa Barbara, CA.

Bradshaw RH, Marchant JN, Meredith MJ, Broom DM (1998). Effects of lavender straw on stress and travel sickness in pigs. J Altern Compl Med 4: 271-275.

Buchbauer G, Jirovetz L, Jaeger W (1991). Aromatherapy: evidence for sedative effects of the essential oil of lavender after inhalation. Z Naturforsch 46: 1067-1072.

Buchbauer G, Jirovetz L, Jaeger W, Plank C, Dietrich H (1993). Fragrance compounds and essential oils with sedative effects upon inhalation. J Pharm Sci 82: 660-664.

Cavanagh HMA, Wilkinson JM (2002). Biological activities of lavender essential oil. Phytother Res 16: 301-308.

Cerny A, Schmid K (1999). Tolerability and efficacy of valerian/ lemon balm in healthy volunteers (a double-blind, placebocontrolled, multicentre study). Fitoterapia 70: 221-228.

de las Cuevas C, Sanz E, de la Fuente J (2003). Benzodiazepines: more 'behavioural' addiction than dependence. J Psychopharmacol (Berl) 167: 297-303.

Diego MA, Jones NA, Field T, Hernandes-Reif M, Schauberg S, Kuhn C et al (1998). Aromatherapy positively affects mood, EEG patterns of alertness and math comutations. Int J Neurosci 96: 217-224.

Elisabetsky E, Brum LF, Souza DO (1999). Aniconvulsant properties of linalool in glutamate-related seizure models. Phytomedicine 6: 107-113. 
Elisabetsky E, Coelho de Souza GP, Dos Santos MAC, Siquieira IR, Amador TA (1995). Sedative properties of linalool. Fitoterapia 46: 407-414.

Fowles DC, Christie MJ, Edelberg R (1981). Publication recommendations for electrodermal measurements. Psychophysiology 18: 232.

Hardy M, Kirk-Smith MD, Stretch DD (1995). Replacement of drug treatment for insomnia by ambient odour. Lancet 346: 701.

Hirst A, Sloan R (2002). Benzodiazepines and related drugs for insomnia in palliative care. Cochrane Database Syst Rev 4: CD003346.

Hongratanaworakit T, Heuberger E, Buchbauer G (2000). Effects of sandalwood oil and $\alpha$-santalol on humans I: inhalation. Poster presentation, 31st ISEO, Hamburg, Germany, book of abstracts, p A-37.

Hongratanaworakit T, Heuberger E, Buchbauer G (2004). Evaluation of the effects of East Indian sandalwood oil and alphasantalol on humans after transdermal absorption. Planta Med 70: 3-7.

Jaeger W, Buchbauer G, Jirovetz L, Fritzer M (1992). Percutaneous absorption of lavender oil from a massage oil. J Soc Cosmet Chem 43: $49-54$.

Jellinek JS (1997). Psychodynamic odor effects and their mechanisms. Cosmet Toiletries 112: 61-71.

Kennedy DO, Wake G, Savelev S, Tildesley NTJ, Perry EK, Wesnes KA et al (2003). Modulation of mood and cognitive performance following acute administration of single doses of Melissa officinalis (lemon balm) with human CNS nicotinic and muscarinic receptor-binding properties. Neuropsychopharmacology 28: 1871-1881.

Kerl S (1997). Zur olfaktorischen Beeinflussbarkeit von Lernprozessen. Dragoco Rep 44: 45-59.

Kirk-Smith M (1995). Possible Psychological and Physiological Processes in Aromatherapy. Aroma '95 Conference Proceedings, Aromatherapy Publications: Brighton. pp 92-103.

Knasko SC (1992). Ambient odor's effect on creativity, mood, and perceived health. Chem Senses 17: 27-35.
Kommission E Monograph (1989) Johanniskraut; Bundesanzeiger Nr. 4302.03.

Lis-Balchin M, Hart S (1999). Studies on the mode of action of the essential oil of lavender (Lavandula angustifolia P. Miller). Phytother Res 13: 540-542.

Oldfield RC (1971). The assessment and analysis of handedness: the Edinburgh inventory. Neuropsychologia 9: 97-113.

Pharmacopoea Europaea (1997). Pharmacopoea Europaea Monograph, 3rd edn. Verlag Oesterreich: Erdnussoel. p 899.

Re L, Barocci S, Sonnino S, Mencarelli A, Vivani C, Paolucci G et al (2000). Linalool modifies the nicotinic receptor-ion channel kinetics at the mouse neuromuscular junction. Pharmacol Res 42: $177-181$.

Schandry R (1989). Lehrbuch der Psychophysiologie, 2nd edn. Psychologie-Verlags-Union: Weinheim.

Schulz H, Jobert M, Huebner WD (1998). The quantitative EEG as a screening instrument to identify sedative effects of single doses of plant extracts in comparison with diazepam. Phytomedicine 5: 449-458.

Stern RM, Ray WJ, Quigley KS (2001). Psychophysiological Recording, 2nd edn. Oxford University Press: New York.

Sugawara Y, Hara C, Tamura K Fujii T, Nakamura K, Masujima T, Aoki T (1998). Sedative effect on humans of inhalation of essential oil of linalool: sensory evaluation and physiological measurements using optically active linalools. Analyt Chim Acta 365: 293-299.

Torii S, Fukada H, Kanemoto H, Miyanchi R, Hamauzu Y, Kawasaki M (1988). Contingent negative variation (CNV) and the psychological effects of odour. In: Van Toller S, Dodd GH (eds). Perfumery - The Psychology and Biology of Fragrance. Chapman \& Hall: London, New York. pp 107-120.

Velden M (1994). Psychophysiologie. Eine kritische Einfuehrung. Quintessenz-Verlags-GmbH: Muenchen.

von Zerssen D, Koeller DM (1976). Bf-S, Befindlichkeitsskala. Beltz: Weinheim.

Yagyu T (1994). Neurophysiological findings on the effects of fragrance: lavender and jasmine. Integr Psychiatry 10: 62-67. 